\title{
Perspectives of Self-Care Experiences of Aging Individuals Living Independently: A Focused Ethnography in the Community Setting
}

\author{
Phatchanun Vivarakanon ${ }^{1}$ \\ ${ }^{1}$ Kramer School of Nursing, Oklahoma City University, Oklahoma, United State of America \\ Correspondence: Phatchanun Vivarakanon, Kramer School of Nursing, Oklahoma City University, Oklahoma, \\ USA. Tel: 1-405-510-8532. ORCID ID: https://orcid.org/0000-0003-1289-1691
}

Received: March 19, 2021 Accepted: April 19, 2021 Online Published: May 8, 2021

doi:10.5539/gjhs.v13n6p81 URL: https://doi.org/10.5539/gjhs.v13n6p81

\begin{abstract}
Globally, aging individuals who live independently facing various problems in maintaining their health and well-being. Understanding the capability of personal well-being is an important consideration in promoting healthy behaviors and lifestyles in aging individuals. This paper aimed to more deeply explore, from self-care experiences of aging individuals living independently in the community, from their perspective. The study used the inductive approach of focused ethnography which is based on Orem's Theory of Self-Care. Data collection consisted of participant observation with field notes and semi-structured interviews with 25 aging individuals living independently in the northern part of Thailand. Three themes were followed as protocol of the data collection plan and used the identification and classification of transcription, coding, and thematic analysis as perspectives of self-care experiences of aging individuals living independently in the community setting: 1) continuing habits of healthcare practices, 2) maintaining positive emotional adaptation, 3) and having reasonable social and life adjustments. These themes exemplified the practice of activities that aging individuals initiated and performed as their daily and routine activities with the intention of maintaining life health and well-being. Consideration of aging individuals living independently self-care experiences assisted nurses and provided greater perspectives in providing actual needs and reduced resources of nursing care and healthcare system.
\end{abstract}

Keywords: self-care experiences, self-care behaviors, aging healthcare experiences

\section{Introduction}

The trend of an aging population is a key consideration in planning and developing successful health and palliative care. In the US, the number of aging individuals over 65 years of age will be more than double over the next 25 years as baby boomers age (Center to Advanced Palliative Care, 2019). Similarly, in Thailand, the latest UN estimation of the number of persons, aged 60 and over was 10.7 million in 2015 and will continue to increase to more than 20 million individuals over the next two decades (Knodel, Teerawichitchinan, Prachuabmoh, \& Pothisiri, 2015; United Nations Population Found, 2020). The total population of aged individuals, living independently and single in Thailand is only $8.8 \%$, while $19 \%$ lived with a spouse (Knodel et al., 2015). The Social and Quality of Life Database System [SQLDS] (2018) indicates that the number of aging individuals living independently in Thailand reaches $1,223,451$, approximately $10.8 \%$ of the total population in 2016 . The data reported that 797,579 of the aging population are women who lived independently, while 425,864 of this population are men (SQLDS, 2018) presented in Appendix A. This population is the target in obtaining data specifically involving the living behaviors as a cross-section that represents the total population.

The number in the aging population; especially, those who live independently is steadily increasing (Knodel et al., 2015; SQLDS, 2018). The latest evidence shows that the majority (96.8\%) of aging individuals living dependently in urban areas have lower levels of self-care ability and which is statistically significant between their self-care ability and academic level, life styles, occupation status, and marital status $(p<0.05)$ (Tabrizi, Behghadami, Saadati, \& Soderhamn, 2018). Noticeably, individual self-care maybe influencing various factors. This could be explained as Dorotha E. Orem stated in the Theory of Self-Care. A conceptual model for nursing practice, the theory of self-care develops as a result of Dorothea E. Orem (Denyes, Orem, \& Bekel, 2001; Riegel, Jarrsma, \& Stomberg, 2012). SCDT explains that all individuals are capable of self-care, consisting of actions and are performed on their own behalf to maintain life, health, and well-being (Denges et al., 2001). This theory shapes an inductive approach where the researcher begins with few preconceptions about self-care experiences in aging individuals who live 
independently. Additionally, it was devised to explain what was seen rather than the other way around based on SCDT. In aging individuals with chronic illnesses, self-care processes such as health promoting practices and managing illnesses have been commonly found (Denyes et al., 2001; Riegel et al., 2012). The previous study claimed that self-care was defined as a fundamental aspect to sustain of health, prevent probable physical issues, and manage of chronic illnesses; similarly, SCDT developed by Orem mentioned (Denyes et al., 2001; Riegel et al., 2012). It notices that self-care implementation influences individuals performing habitual behaviors. However, there are many factors influencing self-care management and plans of aging individuals such as environments, living behaviors, and relationships with others (Alligood, 2017). The previous evidence showed that home-dwelling older individuals without physical limitation were responsible for their daily activities without support from others, while those with physical limitation showed abandoned self-care required assistance to manage their routine activities, especially home-dwelling aging individuals who lived independently (Back \& HEntinen, 2001). In addition, the relationship between aging individuals and caregivers is associated with their self-care and confidence to engage in the aging individual's selfcare in the palliative care setting $(p=.003)$ (Riegel et al., 2009). Thus, it is challenging to deeply understand how aging individuals living independently state their perspectives of self-care experiences using the inductive approach of focused ethnography based on Orem's Theory of Self-care.

An ethnographic approach to qualitative research is adapted for the study. Using the focused ethnographic design to identify and describe perspectives of self-care experiences is conducted to allow for a deep understanding of a particular behavior. Additionally, the approach could support deeper discovery of specific beliefs, experiences, and practices of particular individual self-care as held by individuals. At a minimum in providing suitable support and care based on aging individuals' experiences, healthcare providers and caregivers could reduce their workload, individuals' length-of-stay, and occupancy rates (Aoun et al., 2016). This study purposed to gain deeper exploration, from their perspectives of aging individuals living independently in the community settings, and their self-care experiences.

\section{Methods}

This study used the inductive approach of focused ethnography which is based on Orem's Theory of Self-Care. It identifies and describes perspectives of aging individuals living independently related to their self-care experiences. Semi-structured interview questions focused and provided guidance for the study. These questions were presented in the setting of a community in the northern province of Thailand. The protocol in collecting data through processes of analyzing data was developed as a guideline plan presented in Appendix B. This protocol was used as a guideline in collecting data step-by-step for researcher to strengthen data collected. The researcher was trained in responsible research conduct and issues involving informed consent, ethics, and confidentiality by a pilot study. Starting with the data collection plan, the researcher conducted a pilot test using the same processes of real interviews to understand individuals' thoughts and reactions as well as potential and unforeseen problems. Two or three participants who met the eligibility criteria were recruited, and sign the informed consent. During the interview process, the researcher identified any problems. If it related to participant attritions such as uncomfortable responses and health problems, the researcher made the decision to accept or recruit the participant based on their needs. Follow-up was needed if there were unanswered questions until data saturation. However, when conducting a pilot study, the researcher needed to control consistency of the research design and method to reduce the influence of intervening factors. The researcher needed to be more realistic in sensing problems, and be flexible in dealing with problems from samples, individuals responses, external influences, and role conflicts. Further, procedures of the protocol consistently assisted the researcher in conducting data collected through data report.

\subsection{Setting and Participant}

The proposed research study was conducted in a northern province of Thailand: an area with a population of 61,085 aging individuals living independently, approximately $8.22 \%$ of the total population of 744,945 (SQLDS, 2017). Twenty-five individuals were selected to obtain enough data to sufficiently describe the phenomenon of interest and address the research questions. Criteria, both inclusion and exclusion in selection were key features for potential individuals and were crucial in designing the study. Inclusion criteria included aging individuals, aged 60 or over, who lived independently and those who lived with only a spouse. Participants might or might not have a house to live in, and it was unknown whether they had any support from their children or other relatives. Participants who were diagnosed with at least one chronic illness such as cancer, hypertension (HT), or diabetes with life expectancy of more than a year. Participants had no reported mental health or dementia diagnoses that would prevent them from participating in the interviews. Exclusion criteria included individuals who lived with 
their families for support such as money, housing, and transportation. Participants diagnosed with end-stage diseases and a confirmed prognosis of living less than one year.

\subsection{Research Design}

This study was guided by focused ethnography, which required an understanding of aging individuals living independently in the community within a cultural group. Preconception based on Orem's Theory of Self-Care were devised to explain what was seen rather than the other way around relating to self-care experiences. To gain depth of exploration, semi-structured interview, participant observation, field notes, and video recording were used to conduct data. A semi-structured interview was used to gain understanding of participant words during observation situations. The field note consisted of content, metacommunication, and context along with researcher's responses and reactions to what emerged and transpired in data collection over extended periods of time. Video recorded and transcribed verbatim were used as tools during the three phrases of collecting data.

\subsection{Data Collection}

Data was collected from May 2020 to December 2020 by the author, who face-to-face interviewed participants via video recording. Interview length was 40 to 60 minutes, with $80 \%$ being $45-60$ minutes in length by using semi-structured interview guidelines for exploring the participant's perspectives of self-care experiences in the community settings: a) How do you take care of yourself when you have minor illnesses, stress or anxiety, and do outside activities? b) How do you take care of your emotional and spiritual aspects? And c) Why and How do you make social connections when you lived independently?

The semi-structured interviews were referred to the Theory of Self-Care principle regarding regularly and routine practices and activities which covered a holistic way of physical, psychological, social, and spiritual perspectives. In the process of interview questions, the researcher introduced herself and built a rapport with participants briefly describing the purpose of the interview as well as the use of video recording. The researcher stated a simple question and then moved to in-depth questions regarding self-care experiences.

In addition, the researcher used a method of participant observation by which the researcher joined the insider participant. The participant observation was used during interviewing as a means for a generating more complete understanding of the participant's activities. Immersion was a key element in order to reduce bias; however, the field setting, Lampang Province in northern Thailand, was the place where the researcher lived, and it benefited in understanding aging individual's lifestyles, beliefs, and cultural differences.

In the field setting, the researcher continually kept detailed field notes of all observations made during interviewing and meeting participants. Each participant was given one-on-one observation with different times for meeting and interviewing by video recording. The field notes helped the researcher regarding detailed aspects of discourse. All writing had detailed information, and was transcribed immediately after the meeting.

\subsection{Data Analysis}

Data analysis included the identification and classification of transcription, coding, and thematic analysis. Each transcript was repeated and reviewed for meaningful and significant reporting before conducting coding. The MAXQDA 2020 was used as a coding software program to manage data and conduct analysis. To in-depth understand individuals' views, knowledge, experiences, and values, thematic analysis focused on identifying and describing both implicit and explicit data collection.

\subsection{Methodological Rigor}

Dependability of this study used the inductive approach based on Orem's Theory of Self-care as well as protocol of the data collection plan. To strengthen credibility, the researcher included various branches of trustworthy principles such as building a good rapport, practicing effective communication, and selecting effective databased and access for references. The researcher also used the principle of reflexivity how the researcher and individuals thought, what they plaid attention to, and how they asked questions to increase understanding and describe the individual's cultural or personal group. In addition, the researcher shared purpose and knowledge translation by looking for transferability in non-participant persons in the setting field to share their cultural knowledge and common read on the study. A member check was used to assess the accuracy of the description and solicited their in-depth understanding of data collection. To avoid biases, the researcher immerged own self into the field setting to understand people as an individual with the aim to maintain dependability, credibility, and transferability.

\subsection{Ethics}

Ethical approval was taken from ethical review board of Oklahoma City University procedures and guidelines, including anonymity, participants' privacy, and confidentiality during data collection and publication. Written 
informed consent was received from the participants before collecting data. There were no specific treatments, training courses, or invasive procedures in this study. Approval by the Institutional Review Board (IRB) of Oklahoma City University and a long-term care institution was used to protect individuals' rights as participants.

\section{Results}

\subsection{Participant Data}

The socio-demographic characteristics include age, gender, education, marital, job status, and chronic illnesses of aging individuals living independently were presented in Table 1. The average age of participants was 73.6 years old. Females were the majority gender at $64 \%$ while $36 \%$ were males. Almost half of the participants did not study, whereas $44 \%$ were those who graduated from primary school. The marital status of participants was $44 \%$ while only $4 \%$ was single. Though $76 \%$ of aging individuals were unemployed, $24 \%$ or 6 were employed. All participants had at least one chronic illness, and $24 \%$ had two or more underlying chronic illnesses.

Table 1. Frequencies and percentages of demographic characteristics $(\mathrm{N}=25)$

\begin{tabular}{lrc}
\hline & Frequency & Percent \\
\hline Age (years) & 8 & 32.0 \\
$60-69$ & 13 & 52.0 \\
$70-79$ & 4 & 16.0 \\
$>80$ & 9 & 36 \\
\hline Gender & 16 & 64 \\
$\quad$ Male & 12 & \\
Female & 11 & 48 \\
\hline Education & 2 & 44 \\
No school & 1 & 8 \\
Primary School & 11 & 4 \\
$>$ Middle School & 5 & 44 \\
\hline Marital Status & 8 & 20 \\
Single & & 32 \\
Married & 19 & 76 \\
Divorced & 6 & 24 \\
Wisdom & 19 & 24 \\
\hline Job Status & 6 & \\
Unemployed & & \\
Working & & \\
\hline Chronic Illnesses & & \\
One illness & 12 & \\
$>2$ illnesses & & \\
\hline
\end{tabular}

\subsection{Thematic Analysis}

In collecting actual data, the semi-structured interview; participation observation; filed notes along with video recording indicated that aging individuals who lived independently comfortably shared their habitual self-care experiences. Three themes were found as perspectives of self-care experiences of aging individuals who lived independently in the community settings presented in Table 2 . 
Table 2. Thematic Analysis

\begin{tabular}{|c|c|c|c|}
\hline Sub-Themes & $\begin{array}{l}\text { Continuing habit of } \\
\text { healthcare practices }\end{array}$ & $\begin{array}{l}\text { Maintaining positive } \\
\text { emotional adaptation }\end{array}$ & $\begin{array}{l}\text { Having reasonable social and } \\
\text { life adjustment }\end{array}$ \\
\hline $1^{\text {st }}$ & $\begin{array}{l}\text { Associated with past } \\
\text { experiences and } \\
\text { understanding }\end{array}$ & $\begin{array}{l}\text { Ignore stress and dissatisfied } \\
\text { situation }\end{array}$ & $\begin{array}{l}\text { Keep their social connections } \\
\text { with their own ways }\end{array}$ \\
\hline $2^{\text {nd }}$ & $\begin{array}{l}\text { Adapt their healthcare } \\
\text { management plans into daily } \\
\text { activity and living behaviors }\end{array}$ & $\begin{array}{l}\text { Going to temple, praying, } \\
\text { and doing religious attention }\end{array}$ & $\begin{array}{l}\text { Effortlessly adapted } \\
\text { themselves into current } \\
\text { situation }\end{array}$ \\
\hline $3^{\text {rd }}$ & & & $\begin{array}{l}\text { Keep routines such as } \\
\text { exercising }\end{array}$ \\
\hline
\end{tabular}

\subsubsection{Continuing Habit of Healthcare Practices Theme}

The first semi-structured interview question "How do you take care of yourself when you have minor illnesses, stress or anxiety, and do outside activities?" was used to inquire about perspectives of self-care experiences regarding physical care of their health and illness. Aging individuals living independently in the community tended to make healthcare a habit for their physical and chronic illnesses. Both physical and chronic illness care, were generally based on their past experiences and knowledge. Taking medicine, massaging, and exercising were personal care activities, which aging individuals did when they had minor illnesses.

In the following field note, the seventh participant pointed out, "I do hand exercises in the morning and at night daily because it helps me to relive pain and numbness when I sleep at night. The nurse told me to do when I came to do them when I went to follow up chronic illnesses at the hospital."

Likewise, self-care experiences of chronic illnesses, aging individuals living independently were likely to habitually take medicine as continued care for their chronic illnesses. The seventh participant mentioned, "I treat my illnesses... and go to the hospital to receive medicine every three months. I am still taking hypertension medicine." However, because of living independently and having financial burdens, aging individuals tended to take care of themselves when they were minorly sick; and going to the hospital or clinic was not a first choice for them. As the twelfth participant explained:

I have had hypertension since 2010 and was diagnosed with Herniated Nucleus Pulposus 2 years ago. I wanted to be better from my illnesses. The doctor told me to have surgery, but I think it is a big thing to be admitted into the hospital because my children live in another city and they are busy.

From observations, each participant had their prescribed and herbal medicine, and they knew when to take and use it. During the interview about exercise, participants with and without physical difficulties demonstrated how to do exercise even on the bed. This reflected that aging individuals living independently continually performed habitually self-care on their own behalf in the interest and experience following the Theory of Self-care.

\subsubsection{Maintaining Positive Emotional Adaptation Theme}

The next semi-structured interview question. "How do you take care of your emotional and spiritual needs?" was used to inquire about perspectives of self-care experiences regarding emotional and spiritual perspectives. Aging individuals living independently had fairly positive moods and feelings in regard to loneliness and sickness. Positive adaptation has been found in emotional and spiritual perspectives. Ignoring stress and changing probable behaviors increased emotional strength in aging individuals living independently. In the field note, ten participants tended to pay no attention to unpleasant situation as well as accepted their current sickness and physical decline as changes with age. The eighteenth participant stated, "I try to not think too much and I ignore unpleasant situations because no one can help you except yourself." Similarly, the thirteenth participant claimed, "I like to think positively because it helps me feel good and be physical healthy because I have hypertension and diabetics. I do not want to be sick and have more physical decline."

Self-care experiences with positive emotional adaptation were also related to religious aspects. Almost half of the participants stated the temple, meditation, and praying were well-known as religious ways for aging Buddhist Thais. The fifth participant said, "I like to go to the temple every day except rainy ones. I feel much better and blissful when I go to the temple, also I meet a lot of friends there." The sixteenth participants said: 
I used to go to the temple before, but I have not been there for a long time since I had an accident three years ago. However, I still remind myself that my is a temple. Every night, I pray before I sleep, it helps me feel better even though I cannot go to the temple.

Aging individuals living independently have the risk factors for unpleasant situations although living independently like isolation and depression. From observation, when the researcher talked about living independently, participants' faces showed a little bit of disappointment; however, they were happy to finally think positively. In addition, all participant's homes had a monk statue and religious books. This means that religious aspects are important perspectives for aging individuals for releasing their loneliness, depression, and sadness.

\subsubsection{Having Reasonable Social and Life Adjustment Theme}

The third semi-structured interview question, "Why and How do you take care of social connection when you lived independently?, was used to explore perspectives of self-care experiences regarding social aspect. The majority of aging individuals living independently tended to continually keep social contact with others in the same community. Doing outside activities such as going to the market and visiting with friends were common activities in aging individuals living independently without physical difficulties. The twenty second participant said, "I keep doing activities that I can do around my home. Sometimes, I visit my friends in the market." The eleventh participant said, "When I have available time during the day, I go to talk with my friends who live near my home. We like to talk and share daily living experiences such as how to take care of ourselves, healthcare management, and alternative medicine." The seventh participants said, "I always go out to a market every day. I think I do not feel lonely and it is relaxing when I go to the market because I see a lot of friends and we share information."

However, some aging individuals living independently with physical difficulties do not do outside activities and do not feel lonely because friend's visit. Aging individuals living independently have also accepted their current health status as well as understanding that death was normal. The sixth participant said, "My relatives come to visit as often as they have time. They come to help me sometimes, share food, and talk with me. I feel very blissful that I have strong relationships with my family and other relatives." The fifteenth participant said, "Although my children live in another city, my neighbors who live beside my house continuously come to visit and help me when I need support."

Additionally, because of the insufficient money support, aging individuals living independently continue to work. Aging individuals living independently without physical difficulties and those between 61 and 79 years-old were planting vegetables and making meatball skewers to keep earning. The twenty second participant said:

I actually have keen problems, but I try to plant

some vegetables for sell because the aging subsidy is not

enough for me to live. Everything is so expensive. So, I need to make money for daily living as

much as I can.

The thirteenth participant said, "I do meatball skewers because someone hires me to do it. I think it is easy for me to do and it is good for me to make some money as I get older. I get the subsidy support, but it is not enough for daily living.

Along with field notes and participant observation, the researcher noted that at every interview with participants, most had at least one guest who was a friend and neighbor who lived beside or around them. When the participants answered interview questions, their visitors helped them think and remind them of what activities they did as routines of individual care. In addition, the temple and fresh market were places of social meetings for them. Some participant's home had small to large vegetable pots with the purpose to cook for meals and sell in the fresh market.

\section{Discussion}

In this study, we explored perspectives of self-care experiences of aging individuals who lived independently in the community. Data saturation, was reach by the twenty-second interview, with twenty-five total interviews completed before there were no new information adding into new themes when using thematic analysis. We then focused on how these perspectives reasonably conferred self-care experiences of aging individuals living independently in the community setting. The first theme presented continued habit of healthcare practices of aging individuals living independently in the community settings. Habits of ongoing personal care such as taking medicine, massaging, and exercising were common and usual practices for them, and were associated with past experiences and understanding. As a result, several aging individuals living independently who were concerned with and understood healthcare practices tended to appropriately combine traditional ways to treat their physical decline and chronic illnesses such as taking herbal medicine, massaging, and applying balm. Aging individuals 
living independently who understood herbal medicine were likely to adapt herbal medicine as a part of healthcare routinely to avoid physical decline and regurgitation of chronic illnesses. The perspective of the continued habit of healthcare practices was associated with the theory of self-care that Alligood (2017), Denyes et al. (2001) and Riegel et al. (2012) stated that the practice of self-care activities initiated and performed on their own behalf in the interest of maintaining life, continuing personal development, and well-being because self-care was an action system. Likewise, Wijk and Grimby (2008) claimed that the experience of pain and poor hygiene of aging individuals with chronic illnesses were related to individual's experiences and understanding. Stimulating aging individuals to learn healthcare experiences via eHealth application improved communication and connectivity with healthcare teams (Buck, Model, Riegel, McMillan, \& Bakitas, 2015). This indicated that personal experiences had impacted how aging individuals performed daily activities related to self-care management, as well as appearing that individual's experiences may often be associated with how they prioritize the physical management of symptoms. The experiences and understanding of physical healthcare primarily strikes nurses in planning and promoting healthcare management of aging individuals living independently into daily activity and living behaviors. For home care services, nurses could plan to educate physical self-care practices to aging individuals as a primary strategy on their own behalf for their interest to maintain life as a normal, be healthful functioning, and continue personal development and well-being. Nurses could further plan promoting physical self-care practices via electronic platforms such as online, application, and e-book.

The second theme presented aging individuals living independently having positive emotional and spiritual adaptation. Well-behaved emotional adjustment as aged older, aging individuals living independently were likely to ignore dissatisfied situations such as feeling of lonely, stress, and anxiety. When aging individuals living independently felt stress and anxiety because of financial burden, they primarily solved problems by themselves first. Asking for help and support regarding health assistance and money found as a second though for them. Although, aging individuals living independently had health problems, money issues, and lived independently that stimulated them to think and stress, they tended to solve first and play no attention to unpleasant situation as well as accepted their current sickness, physical decline, and changes with age. This was associated with their stages of growth and development in psychology and moods as one of characteristics in theory of self-care developed by Orem (Alligood, 2017; Denyes et al., 2001). How aging individual living independently were well-behaved in maintaining positive emotional adaptation showed a growth and development in mindset and moods following the theory of self-care. Although, the latest evidence claimed that the loneliness was a key indicating mental health problems, including both anxiety and depressive symptoms, aging individuals had positive emotion as aged increased (Wijk \& Grimby, 2008). This noticed that why aging Thai individuals living independently were less feeling of loneliness. This was because in Thai culture, family members or relative commonly lived near aging individuals although aging individuals were living independently in their house. Feeling of support from others relieved feeling of loneliness in aging individuals living independently. Similarly, among the Northern, Western, and Eastern European countries had strong family networks with aging individuals who lived independently by living close with aging persons in the same area, street, or neighborhood (Widberg, Wiklund, \& Klarare, 2020). This explained why aging individuals living independently did not feel lonely. Further, the positive emotional adaptation such as feeling of peacefulness, sense of value, and unavoidable suffering of aging individuals living independently were associated with religion perspective. Going to temple, prying, and doing religious attention had been generally found in aging Thai individuals living independently. The latest study beside claimed that feeling more religious as aged older mostly found in aging individuals than other age group (Bekhet \& Zauszniewski, 2014). The religion aspect influenced how aging individuals expected their later life, especially in Buddhism culture, a temple was a place to increase emotional upright for aging individuals (Djundeva, Dykstra, \& Fokkema, 2019). Thus, both living styles and religion aspects found as self-care experiences of emotional aspect of aging individuals living independently. In nursing practices, this theme illustrated that although aging individuals living independently in the community settings tended to be well-behave in having positive emotional adaptation, nurses should still provide psychological support to encourage individuals' self-care as the description in theory of self-care stated.

The last theme of self-care experiences resulted in social and living perspectives. Fairly social and life adjustment were found as self-care management to keep individuals in touch and need to continue daily life. Either aging individuals with or without physical difficulties who living independently had continually kept their social connection with friends, relatives, and communities in their own ways. Aging individuals living independently without physical difficulties kept their social connection by maintaining their outside activities such as going food shopping, visits in the neighbors, and going to temple. On the other hand, those with physical difficulties had a spouse, friends, relatives, and neighbors to take care, talk, and visit with via phone call. The perspective of having 
reasonable social and life adjustment following Orem's stated that maintaining of balance between solitude and social interaction and promoting of human functioning within social groups resulted in the human desire to be normal (Alligood, 2017; Denyes et al., 2001). This noticed that the need of assistance because of the limitation for actions in care themselves resulted in the desire to make life-sustaining and function-regulating inputs (Alligood, 2017; Denyes et al., 2001). However, feeling of being independent regularly found in aging individuals who lived independently caused stress, depression, and lonely, they felt cut off isolated when they continually kept their social connection (Djundeva et al., 2019; Knodel et al., 2015). Additionally, aging individuals living independently were fairly life adjustment, meant they effortlessly adapted themselves into their current situations. Because of insufficient money support, aging individuals living independently continually kept working to maintain daily life and sustain their health status. The support money from Thai government was not enough to survive for aging individuals (Djundeva et al., 2019). As a result, aging independently aged 61 to 70 years-old had continually been working from home such as planting vegetables and making meatball skewers. Further, by the reason of the need to be active aging and no decline, aging individuals living independently kept themselves to be active by keeping daily routine and working as exercising because it was good for their health, releasing stress, and sustained social connection in belonging in the community. Likewise, the previous evidence claimed that being active aging reduced risks of injury and increased productivity as well as health benefits for aging working (Gijsberts, Liefbroer, Otten, \& Olsman, 2019). Thus, being fairly social and life adjustment found as one of the important self-care experiences in aging individuals who lived independently in maintaining both physical and psychological well-being. In homecare services or primary care services, nurses could plan a social support from relevant organizations and government in order to assist sufficient money and working for aging individuals, especially who lived independently. This was because maintaining of balance between social interaction and support found as self-care practice related to social dimension and it was significantly increased quality of life.

\section{Conclusion}

The perspectives of self-care experiences: 1) the continued habit of healthcare practice, 2) maintained positive emotional adaptation, and 3) reasonable social and life adjustment, for these aging individuals living independently in the community setting, evidently illustrated how they behaved personal care daily. Consideration of their self-care experiences of aging individuals living independently assisted nurses providing greater perspectives in providing actual needs and reduced resources of health and nursing care systems.

Recommendation for this study was to further explore a specific dimension of perspectives of self-care experiences to understand in-deptly what aging individuals experienced in each self-care perspective. Researchers could further decide whether to apply another nursing theory or theoretical framework to shape a new framework in other settings or other age groups. Applying to promote healthcare services including health promotion, prevention, treatment, and rehabilitation to both aging individuals who were admitted to the hospital and those who lived in the community setting could be recommended.

\section{Acknowledgements}

Dr.Elizabeth Diener, Dr.Dia Campbell-Detrixhe, and Dr. Cheryl Frutchey were accountable for conception; design; acquisition, drafting.

Nicha Chiayasit contributed to coordinate the participants as well as set a video recording in the field settings.

Thanks for personal assistance for important intellectual content, prepared final manuscript to be published.

\section{Competing Interests Statement}

The authors declare that there are no competing or potential conflicts of interest.

\section{References}

Alligood, M. R. (2017). Nursing Theorists and their Work. Elsevier Health Sciences. Greenville, North Carolina.

Backman, K., \& Hentinen, M. (2001). Factors associated with the self-care of home-dwelling elderly. Scandinavian Journal of Caring Sciences, 15(3), 195-202. https://doi.org/10.1046/j.1471-6712.2001.00007.x

Bekhet, A. K., \& Zauszniewski, J. A. (2012). Mental health of elders in retirement communities: Is loneliness a key factor?. Archives of Psychiatric Nursing, 26(3), 214-224. https://doi.org/10.1016/j.apnu.2011.09.007

Buck, H. G., Mogle, J., Riegel, B., McMillan, S., \& Bakitas, M. (2015). Exploring the relationship of patient and informal caregiver characteristics with heart failure self-care using the actor-partner interdependence model: Implications for outpatient palliative care. Journal of Palliative Medicine, 18(2), 1026-1032. https://doi.org/10.1089/jpm.2015.0086 
Center to Advanced Palliative Care. (2014). Palliative care facts and stats. Retrieved from https://media.capc.org/filter_public/68/bc93c7-14ad-4741-9830-8691729618d0/capc_press-kit.pdf

Denyes, M. J., Orem, D. E., \& Bekel, G. (2001). Self-care: A foundational science. Nursing Science Quarterly, 14(1), 48-54. https://doi.org/10.1177/089431840101400113

Djundeva, M., Dykstra, P. A., \& Fokkema, T. (2019). Is living alone aging alone? Solitary living, network, types, and well-being. The Journals of Gerontology: Series B, 74(8), 1406-1415. https://doi.org/10.1093/geronb/gby119

Gijsberts, M. J. H., Liefbroer, A. I., Otten, R., \& Olsman, E. (2019). Spiritual care in palliative care: A systematic review of the recent European literature. Medical Science, 7(2), 25. https://doi.org/10.3390/medsci7020025

Knodel, J., Teerawichaitchinan, B., Prachuabmoh, V., \& Pothisiri, W. (2015). The situation of Thailand's older population: An update based on the 2014 survey of older persons in Thailand. Retrieved from https://www.psc.isr.umich.edu/pubs/pdf/rr15-847.pdf

LeBlanc, R. G., \& Jacelon, C. S. (2018). Self-care among older people living with chronic conditions. International Journal of Older People Nursing, 13(3), 12191. https://doi.org/10.1111/opn.12191

Mucci, N., Tommasi, E., Giorgi, G., Taddei, G., Traversini, V., Fioriti, M., \& Arcangeli, G. (2019). The working environment as a platform for the promotion of active aging: An Italian overview. The Open Psychology Journal, 12(1). https://doi.org/10.2174/1874350101912010020

Phenwan, T., Peerawong, T., \& Tulathamkij, K. (2019). The meaning of spirituality and spiritual well-being among Thai breast cancer patients: A qualitative study. Indian Journal of Palliative Care, 25(1), 119.

Riegel, B., Jaarsma, T., \& Stromberg, A. (2012). A middle-range theory of self-care of chronic illness. Advances in Nursing Science, 35(3), 194-204. https://doi.org/10.1097/ans.0b013e31826b1ba

Riegel, B., Moser, D. K., Anker, S. D., Appel, L. J., Dunbar, S. B., Grady, K. L., ... \& Whellan, D. J. (2009). State of the science: Promoting self-care in persons with heart failure: A scientific statement from the American Heart Association. Circulation, 120(12), 1141-1163.

Social and Quality of Life Database System. (2018). Number and Percentage of Elderly By occupancy, 2002-2016. Retrieved from http://social.nesdb.go.th/SocialStat/StatReport_Final.aspx?reportid=3501\&template= 2R1C\&yeartype $=$ M\&subcatid $=29$

United Nations Population Found. (2017). Aging. Retrieved from https://www.unfpa.org/ageing

Tabrizi, J. S., Behghadami, M. A., Saadati, M., \& Soderhamn, U. (2018). Self-care ability of older people living in urban areas of northwestern Iran. Iranian Journal of Public Health, 47(12), 1899.

Widberg, C., Wiklund, B., \& Klarare, A. (2020). Patients' experiences of eHealth in palliative care: An integrative review. BMC Palliative Care, 19(1), 1-14. https://doi.org/10.21203/rs.3.rs-37535/v1

Wijk, H., \& Grimby, A. (2008). Needs of elderly patients in palliative care. American Journal of Hospice and Palliative Medicine, 25(2), 106-111. https://doi.org/10.1177/1049909107305646

\section{Notes}

Note 1. This a numbers and percentages of aging population classified by living behavior styles, 2002-2007, Thailand.

Note 2. Protocol of data collection plan. 


\section{Appendix A}

Table 1. Numbers and percentages of aging population classified by living behavior styles, 2002-2007, Thailand

\begin{tabular}{llcccc}
\hline & & $\mathbf{2 0 0 2}$ & $\mathbf{2 0 0 7}$ & $\mathbf{2 0 1 4}$ & $\mathbf{2 0 1 7}$ \\
\hline Living Behaviors & & $5,969,030$ & $7,020,959$ & $10,014,705$ & $11,312,447$ \\
& Live Alone & 373,515 & 538,005 & 875,998 & $1,223,451$ \\
& With Family & $5,595,515$ & $6,482,954$ & $9,138,707$ & $10,008,996$ \\
\hline Living Behaviors (\%) & & 100 & 100 & 100 & 100 \\
& Live Alone & 6.3 & 7.7 & 8.7 & 10.8 \\
& With Family & 93.7 & 92.3 & 91.3 & 89.2 \\
\hline
\end{tabular}

Source: Social and Quality of Life Database System Report (2017).

\section{Appendix B}

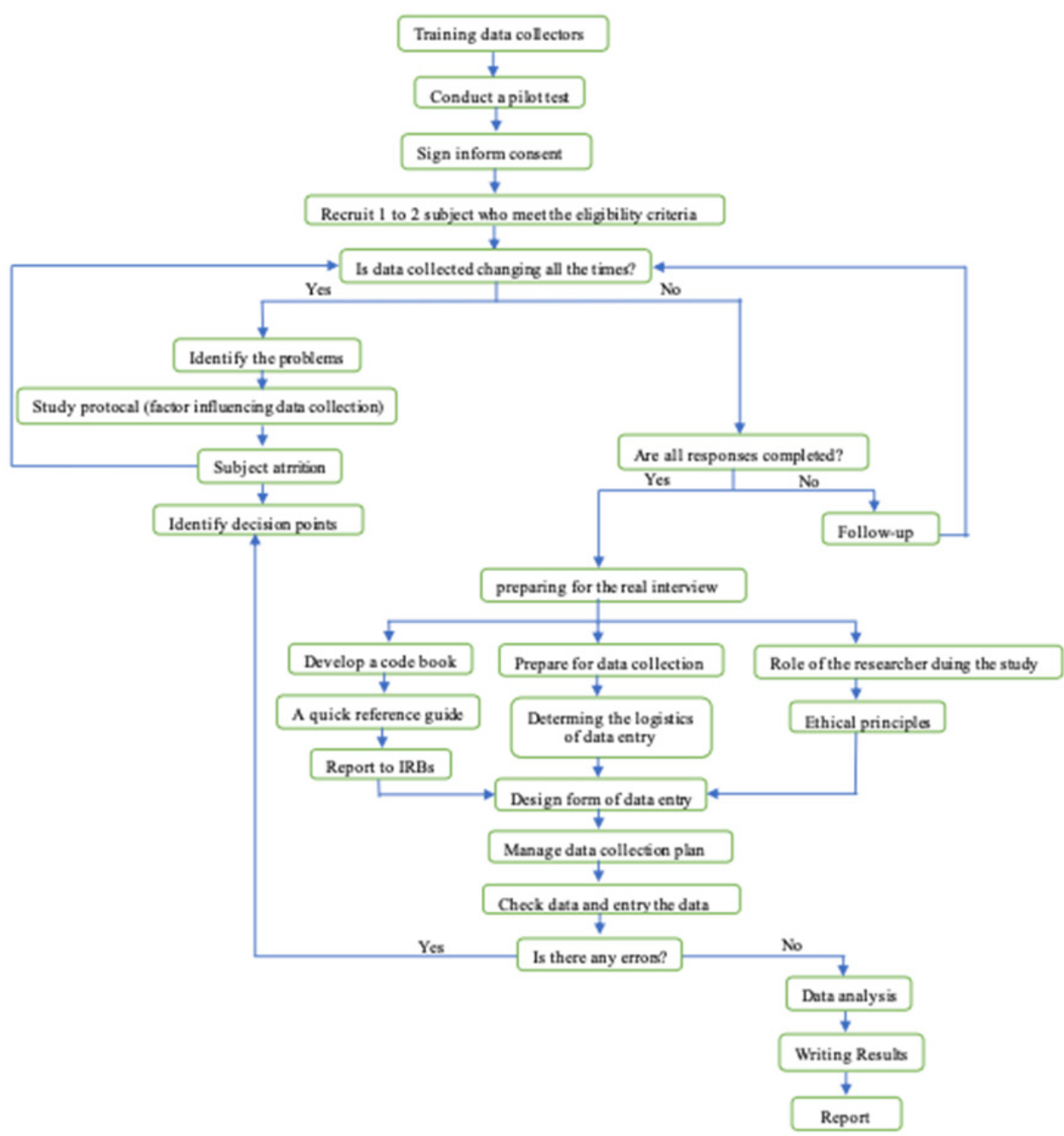

\section{Copyrights}

Copyright for this article is retained by the author(s), with first publication rights granted to the journal.

This is an open-access article distributed under the terms and conditions of the Creative Commons Attribution license (http://creativecommons.org/licenses/by/4.0/). 